\title{
O uso de videogames, jogos de computador e internet por uma amostra de universitários da Universidade de São Paulo
}

\author{
The use of video, computer and internet games by a sample \\ of college students from the University of São Paulo
}

Fernanda Tomie Icassati Suzuki', Marcelo Vieira Matias', Maria Teresa Araujo Silva', Maria Paula Magalhães Tavares de Oliveira'

\section{RESUMO}

Objetivo: Avaliar uso de jogos eletrônicos (videogames, jogos de computador e internet) em uma amostra de universitários. Método: Um questionário a respeito de comportamentos relacionados ao uso de jogos eletrônicos, contendo a escala Problem Videogame Playing (PVP), foi aplicado em 100 alunos da Universidade de São Paulo (USP). Resultados: A maioria (83\%) relatou ter jogado no último ano, dentre a qual 81,9\% eram homens, 51,8\% jogavam de 1 a 2 horas por sessão; 74,4\% afirmaram que jogar não interfere em seus relacionamentos sociais e 60,5\%, que o uso de jogos violentos não influencia sua agressividade. Os estudantes dividiram-se entre jogadores ocasionais e frequentes, diferenciando-se por duração de cada sessão, jogo preferido, motivação para jogar, e influência do jogo na vida social. Cerca de 5\% relataram só parar de jogar quando interrompidos, normalmente jogar mais de 4 horas por sessão e se relacionar mais com amigos virtuais, sugerindo maior envolvimento com a atividade. Na escala PVP, 15,8\% da amostra preencheu mais da metade dos itens, indicando consequências adversas associadas ao uso dos jogos eletrônicos. Conclusão: Observou-se que o uso de jogos eletrônicos é comum entre os estudantes da USP e que uma parcela apresenta problemas relacionados ao excesso de jogo.

\section{ABSTRACT}

Objective: To evaluate the use of electronic games (video games, computer and internet games) in a sample of college students. Method: A questionnaire on behaviors related to the use of electronic games, including the Problem Videogame Playing (PVP) scale was filled in by 100 students from University of São Paulo (USP). Results: The majority (83\%) reported playing electronic games within the last year, among which, 81,9\% were men, 51,8\% played between 1 and 2 hours per session; 74,4\% reported that the practice of electronic games does not interfere on their social relationships and 60,5\% that the use of violent games does not have an effect over their animosity. The sample was divided into occasional players and frequent ones, differing in terms of duration of session, preferred games, motivation for playing and influence of the game

1 Universidade de São Paulo (USP), Instituto de Psicologia.

Recebido em

30/7/2009

Aprovado em

3/9/2009
Endereço para correspondência: Fernanda Tomie Icassati Suzuki

Instituto de Psicologia da Universidade de São Paulo

Departamento de Psicologia Experimental

Laboratório de Psicofarmacologia

Av. Prof. Mello Moraes, 1721, Cidade Universitária - 05508-030 - São Paulo, SP

Telefone: (55 11) 3091-4176

E-mail: fernanda.suzuki@usp.br 


\section{Keywords}

Video game, electronic games, addiction, college students. in their social life. About 5\% reported to stop playing only when interrupted, normally play more than 4 hours by section and have more virtual relationships and friends, suggesting more involvement with the activity. As to the PVP scale, 15,8\% of the sample met 5 itens or more, indicating adverse consequences associated to the use of electronic games. Conclusion: It was noticed that the use of electronic games is common among USP students and a part of them present problems related to excessive gaming.

\section{INTRODUÇÃO}

Com o crescimento da indústria de jogos eletrônicos, como videogames e jogos para computador e internet, muito se especula a respeito da influência de tais jogos no comportamento do jogador, havendo inclusive debate sobre o potencial adictivo do jogo. De modo geral, podemos classificar esses jogos como um tipo de mídia condizente com a atualidade; são atividades que contam cada vez mais com avanços tecnológicos, têm uma estética própria bem desenvolvida, possibilitam novos tipos de interação presencial ou virtual e oferecem uma contingência de rapidez entre estímulo e resposta. Além disso, apresentam uma grande diversidade, desde jogos que requerem atividade eminentemente sensório-motora, até aqueles que exigem do jogador grande capacidade estratégica e de entendimento de regras. É cada vez mais frequente a presença dos jogos eletrônicos em atividades de lazer, no desenvolvimento de habilidades e em atividades didáticas. Além disso, o fenômeno dos jogos eletrônicos tem estreita relação com o uso da internet, uma vez que grande parcela dos jogos existentes pode ser praticada por essa via.

O uso excessivo de videogames e jogos de computador e internet, doravante chamados de jogos eletrônicos, é um comportamento que tem sido estudado sob o prisma dos comportamentos adictivos, tal como o jogo patológico, demonstrando similaridades com drogadicções. A presente pesquisa teve como objetivo investigar a prática desses jogos eletrônicos (excluindo jogos de azar) e eventuais consequências desse hábito entre estudantes universitários brasileiros.

Em estudo conduzido sobre uma emergente adicção em estudantes na década de 80, Soper e Miller ${ }^{1}$ argumentaram que jogos eletrônicos são potencialmente adictivos e bastante distintos de qualquer outra dependência comportamental. Visando estudar o uso de videogames em crianças em idade escolar, Phillips e Griffiths² observaram diferença significativa de gênero: meninos eram 1,4 vez mais propensos a admitir que jogavam do que as meninas; jogavam mais tempo por sessão, mais vezes por semana e assumiam mais que deixavam de fazer lição de casa para jogar. Sete e meio por cento das crianças que participaram do estudo apresentaram problemas relacionados ao jogo.
Os autores se questionaram se esse perfil persistiria depois da adolescência e se existiria relação com outros comportamentos adictivos. Utilizando escala adaptada dos critérios para diagnóstico de jogo patológico do Manual Diagnóstico e Estatístico de Transtornos Mentais (DSM III-R), Griffiths e Hunts ${ }^{3}$ realizaram uma pesquisa com 387 adolescentes americanos, de 12 a 16 anos, visando estimar a prevalência de dependência em jogos de computador. A análise dos resultados indicou que 19,9\% da amostra preenchia critérios de dependência e relatou ter começado a jogar significativamente mais cedo do que o restante da amostra. Além disso, reportou-se que meninos jogam mais que meninas e que possuíam maior probabilidade de serem classificados como dependentes. Visando um instrumento claro e preciso para investigar problemas relacionados ao uso excessivo de jogos eletrônicos, Salguero e Morán 4 propuseram a escala Problem Video Game Playing (PVP), elaborada a partir de revisão dos critérios diagnósticos para dependência de drogas e para jogo patológico do DSM-IV. Através de análise fatorial, verificaram que os 9 itens da escala PVP mediam um único construto e que alta pontuação na escala foi correlacionada com alta frequência de jogo; média alta de tempo jogado; maior tempo jogado em uma única sessão; percepção do próprio participante e de seus familiares quanto a um possível uso excessivo dos jogos eletrônicos; além de pontuação alta na Severity of Dependence Scale. Os autores concluíram que o jogo excessivo está associado a uma série de fatores que lembram síndrome de dependência.

Outro tema importante que tem chamado a atenção é a possível relação entre jogos eletrônicos e violência. Discute-se a influência de jogos eletrônicos sobre o comportamento do jogador, questionando-se a probabilidade de jogos com temas violentos induzirem seus usuários a emitir comportamentos violentos. Revisões de literatura indicam resultados divergentes. Alguns estudiosos afirmam que videogames com conteúdo violento têm relação com um aumento de comportamentos, pensamentos e afetos violentos, aumento de excitação fisiológica e diminuição de comportamentos pró-sociais ${ }^{5}$. Outros atentam para o fato de que a suposição de que jogos com conteúdo violento levam a conduta violenta pode estar baseada na ideia de que, ao jogar, o indivíduo identifica-se com o personagem, libertando-se das amarras sociais que o impediam de entrar 
em um estado selvagem de violência ${ }^{6}$. Outros autores, por sua vez, lançam a hipótese de que a prática de jogos eletrônicos seria válvula de escape de pensamentos e condutas agressivas?. No entanto, há quem sustente a tese de que o jogo violento pode não ter uma consequência direta na agressividade do jogador, porém, pode provocar hostilidade dele com o mundo a sua volta ${ }^{8}$. O jogo violento poderia estimular o indivíduo a imaginar que há riscos e perigos escondidos pelo mundo inteiro, diminuir sua capacidade de julgamento e provocar ideias agressivas? ${ }^{9}$.

A relação entre videogame e relações sociais é outro aspecto interessante que vem sendo objeto de estudo. O uso de jogos eletrônicos tem sido associado a dificuldades de socialização. O termo "amizade eletrônica" foi cunhado para designar esse tipo de relação que alguns jogadores estabelecem, ao envolver-se no jogo em detrimento de manter relações com outras pessoas ${ }^{10}$. Seriam indivíduos que apresentam dificuldades sociais ou emocionais, que utilizam os jogos para escapar, muitas vezes substituindo interações sociais da vida real pela vida virtual, que provocaria menos ansiedade ${ }^{11-13}$. No entanto, esse tema é controverso, uma vez que outros estudos relatam relação positiva entre indicadores de saúde psicossocial e grupos de jogadores, em comparação a grupos de não jogadores ${ }^{8,14}$. Há quem afirme que os jogos representam na verdade uma forma alternativa de socialização e colaboração para a maioria das pessoas ${ }^{15}$.

É interessante notar que a maioria das pesquisas nessa área estuda crianças e adolescentes. Poucos são os estudos que investigam os hábitos de universitários em relação aos jogos eletrônicos, não havendo nenhum estudo brasileiro. A presente pesquisa pretendeu realizar um estudo exploratório com essa população, investigando a prática de jogos eletrônicos e suas eventuais consequências, incluindo questões relacionadas à socialização e relação com a violência possivelmente induzida por alguns tipos de jogos.

\section{MÉTODO}

Trata-se de um estudo descritivo de corte transversal. A amostra da pesquisa foi constituída por 100 estudantes da Universidade de São Paulo. Para se submeter à pesquisa, os estudantes leram e assinaram um termo de consentimento livre e esclarecido. Foi utilizado um questionário, anônimo e de autopreenchimento, no qual constavam 18 perguntas sobre comportamentos relacionados ao uso de jogos eletrônicos (videogames, jogos de computador e internet) e 9 questões que compõem a escala Problem Video Game Playing (PVP) ${ }^{4}$, traduzida para o português para o presente estudo. A coleta de dados foi realizada na Universidade de São Paulo. Foram abordados 135 estudantes, sendo que 35 recusaramse a participar do estudo. Os questionários foram aplicados em horário de almoço, nos quatro restaurantes da universida- de, de forma a permitir uma amostra diversificada, composta por estudantes de diferentes cursos. Os participantes foram abordados pelos pesquisadores, de forma aleatória, na entrada ou saída dos restaurantes. Foram instruídos a responder às perguntas do questionário e, em caso de dúvida, consultar a pessoa responsável pela pesquisa ou deixar a questão em branco. Os questionários respondidos e os termos de consentimento foram depositados em urnas.

Para a análise dos resultados foi utilizado o programa estatístico Statistical Package for the Social Sciences (SPSS). Para fins de comparação, os entrevistados foram divididos em dois grupos, conforme a frequência com que jogaram videogames. Aqueles que jogaram até uma vez por semana foram classificados como jogadores ocasionais e os que jogaram com frequência maior que isso, como jogadores frequentes. Outra análise foi realizada de acordo com a pontuação na escala PVP. Os participantes que responderam positivamente a 5 ou mais itens da escala foram agrupados e chamados de prováveis jogadores dependentes de videogame, e os demais, agrupados e chamados de jogadores sociais. Devido ao limitado número de participantes, em muitas questões, a comparação não foi possível. Na comparação de duas variáveis foi utilizado o teste exato Fisher e, na presença de três ou mais variáveis, foi utilizado o teste de qui-quadrado de Pearson. Para verificar possíveis correlações foi utilizado o coeficiente de Spearman. Foi considerado $p$ menor ou igual a 0,05 para significância estatística. Todas as provas foram bicaudais.

\section{RESULTADOS}

As características sociodemográficas da amostra são apresentadas na Tabela 1. A média da idade encontrada foi de $21,43$ anos ( $d p=3,59)$. A maioria dos estudantes abordados (83\%) revelou que jogou jogos eletrônicos nos últimos 12 meses e observou-se que, dentre esses, havia uma predominância significativa de homens $(p<0,001)$.

Os dados que seguem referem-se à parcela da amostra que jogou jogos eletrônicos nos últimos 12 meses ( $n=83$ ).

A Figura 1 apresenta a frequência com que os estudantes relataram jogar jogos eletrônicos.

Aproximadamente metade dos estudantes $(51,8 \%)$ relatou que jogava, em média, de 1 a 2 horas em cada sessão, sendo que 25,3\% afirmaram jogar até 1 hora, 18,1\% de 2 a 3 horas e 4,8\% afirmaram que jogam 3 horas ou mais. Comparando os jogadores, observou-se que aqueles classificados como jogadores frequentes relataram ficar mais horas em cada sessão do que jogadores ocasionais $\left(X^{2}=9,363\right.$, g.l. $=2$, $p<0,01)$. Questionados sobre o maior tempo que já jogaram videogame em uma única sessão, a mediana obtida foi de 5,75 horas, sendo que um participante afirmou ter jogado por 72 horas consecutivas. 
Tabela 1. Características sociodemográficas dos estudantes universitários de acordo com a prática de jogos eletrônicos

\begin{tabular}{|c|c|c|c|c|}
\hline & \multicolumn{2}{|c|}{$\begin{array}{l}\text { Estudantes que jogaram nos } \\
\text { últimos } 12 \text { meses ( } n=83 \text { ) }\end{array}$} & \multicolumn{2}{|c|}{$\begin{array}{l}\text { Amostra total } \\
(n=100)\end{array}$} \\
\hline & $\mathrm{n}$ & $\%$ & $\mathrm{n}$ & $\%$ \\
\hline \multicolumn{5}{|l|}{ Sexo } \\
\hline masculino & 68 & 81,9 & 72 & 72,0 \\
\hline feminino & 15 & 18,1 & 28 & 28,0 \\
\hline \multicolumn{5}{|l|}{ Idade } \\
\hline 17-19 anos & 33 & 39,8 & 37 & 37,0 \\
\hline $20-22$ anos & 32 & 38,6 & 37 & 37,0 \\
\hline 23-25 anos & 10 & 12,0 & 14 & 14,0 \\
\hline $26-28$ anos & 5 & 6,0 & 6 & 6,0 \\
\hline 29-31 anos & 0 & 0,0 & 2 & 2,0 \\
\hline $32-35$ anos & 2 & 2,4 & 3 & 3,0 \\
\hline \multicolumn{5}{|l|}{ Curso } \\
\hline exatas & 45 & 54,2 & 51 & 51,0 \\
\hline humanas & 18 & 21,7 & 20 & 20,0 \\
\hline biológicas & 15 & 18,1 & 23 & 23,0 \\
\hline \multicolumn{5}{|l|}{ Ano do Curso } \\
\hline $1^{0}$ & 29 & 34,9 & 38 & 38,0 \\
\hline $2^{\circ}$ & 25 & 30,1 & 25 & 25,0 \\
\hline $3^{0}$ & 3 & 3,6 & 3 & 3,0 \\
\hline $4^{0}$ & 10 & 12,0 & 10 & 10,0 \\
\hline $5^{\circ}$ ou mais & 1 & 1,2 & 4 & 4,0 \\
\hline pós-graduação & 9 & 10,8 & 14 & 14,0 \\
\hline \multicolumn{5}{|l|}{ Situação de trabalho } \\
\hline período integral & 3 & 3,6 & 3 & 3,0 \\
\hline meio período & 16 & 19,3 & 20 & 20,0 \\
\hline ocasionalmente & 15 & 18,1 & 16 & 16,0 \\
\hline não trabalha & 49 & 59,0 & 61 & 61,0 \\
\hline
\end{tabular}

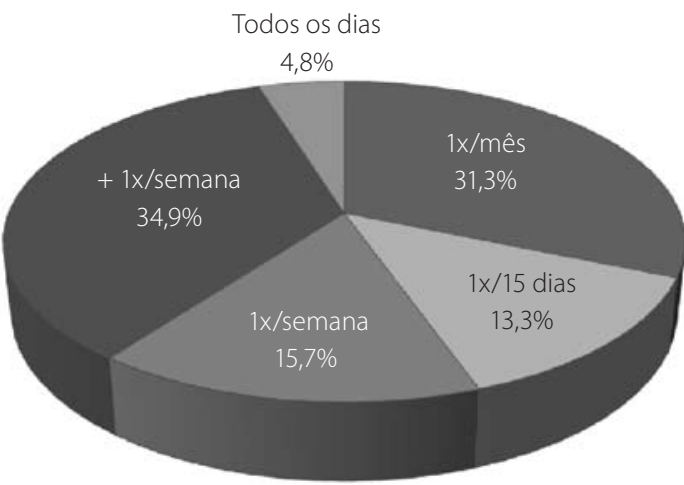

Figura 1. Frequência de prática de jogos eletrônicos pelos universitários nos últimos 12 meses $(n=83)$.

A Figura 2 mostra os tipos de jogos eletrônicos que os estudantes costumam jogar. Observou-se que os jogos de ação e de estratégia foram os únicos assinalados por mais da metade da amostra. Comparando-se os dois grupos, temos que jogadores frequentes costumam jogar mais jogos de estratégia $(p<0,000)$, Role Playing Games (RPG) $(p<0,01)$ e jogos relacionados a esportes $(p<0,05)$ do que os jogadores ocasionais.

Em relação aos períodos em que jogam videogames, a maioria dos participantes $(74,4 \%)$ joga à noite, $40,2 \%$ jogam à tarde, 29,3\% jogam de madrugada e 11\% jogam pela manhã. O local de jogo mais frequente foi o quarto $(55,4 \%)$, seguido pela sala $(38,6 \%)$, casa de conhecidos $(28,9 \%)$, trabaIho/faculdade $(16,9 \%)$ e lanhouses (6\%). Nenhum desses fatores diferenciou jogadores frequentes de ocasionais.

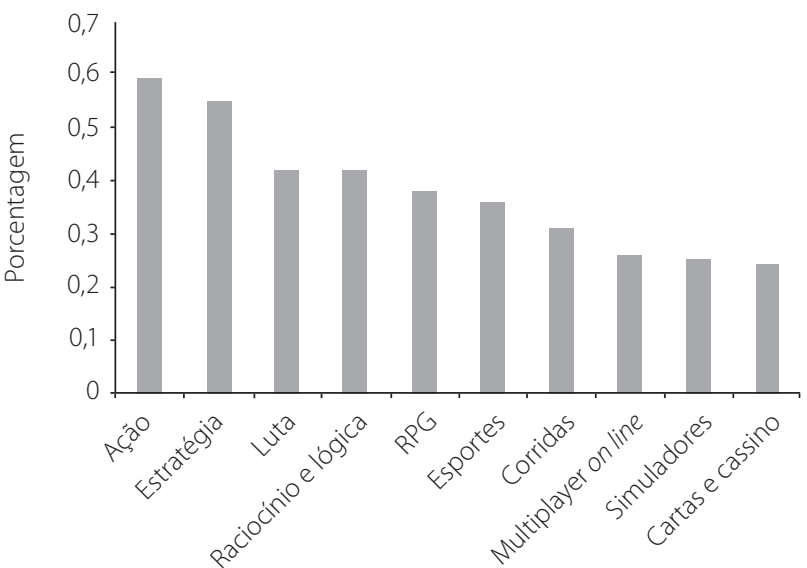

Tipo de jogo

Figura 2. Jogos eletrônicos praticados pelos universitários $(n=83)$.

Com relação ao autocontrole sobre o jogo, 95,2\% dos participantes afirmaram que conseguiam parar de jogar sozinhos, ou seja, que não precisavam que outras pessoas os interrompessem.

Os principais aspectos que motivam o participante a jogar videogame são apresentados na Tabela 2.

Foi encontrada correlação entre jogadores que afirmaram jogar jogos de estratégia e do tipo RPG e aqueles que listaram o desafio de superar etapas entre os motivos que os levam a jogar ( $r=0,03, p<0,01$ e $r=0,25, p<0,05$, respectivamente).

Tabela 2. Aspectos que motivaram os estudantes universitários a jogar jogos eletrônicos de acordo com frequência de jogo

\begin{tabular}{|c|c|c|c|c|c|c|c|}
\hline & \multicolumn{2}{|c|}{$\begin{array}{l}\text { Ocasionais } \\
(n=37)\end{array}$} & \multicolumn{2}{|c|}{$\begin{array}{l}\text { Frequentes } \\
\qquad(n=46)\end{array}$} & \multicolumn{2}{|c|}{$\begin{array}{l}\text { Total } \\
(n=83)\end{array}$} & \multirow[t]{2}{*}{ p } \\
\hline & n & $\%$ & n & $\%$ & $\mathrm{n}$ & $\%$ & \\
\hline Possibilidade de relaxar & 27 & 73,0 & 41 & 89,1 & 68 & 81,9 & 0,085 \\
\hline Prazer no desafio de superar etapas & 19 & 51,4 & 36 & 78,3 & 55 & 66,3 & 0,019 \\
\hline Poder jogar com amigos & 14 & 37,8 & 34 & 73,9 & 48 & 57,8 & 0,002 \\
\hline Observar a arte do jogo & 8 & 21,6 & 18 & 39,1 & 26 & 31,3 & 0,101 \\
\hline Assumir novos papéis & 5 & 13,5 & 14 & 30,4 & 19 & 22,9 & 0,113 \\
\hline $\begin{array}{l}\text { Prazer no controle do mundo } \\
\text { simulado }\end{array}$ & 6 & 16,2 & 12 & 26,1 & 18 & 21,7 & 0,300 \\
\hline Jogar com amigos virtuais & 3 & 8,1 & 10 & 21,7 & 13 & 15,7 & 1,000 \\
\hline Extravasar a raiva & 2 & 5,4 & 5 & 10,9 & 7 & 8,4 & 0,453 \\
\hline Outros & 3 & 8,1 & 4 & 8,7 & 7 & 8,4 & 1,000 \\
\hline
\end{tabular}


Questionados sobre a influência de jogos violentos sobre seu comportamento, a maioria dos estudantes $(60,5 \%)$ relatou sentir-se indiferente após jogar um jogo violento, enquanto que $8,6 \%$ disseram que se sentem mais relaxados e 7,4\% assumiram que se sentem mais agressivos. Em relação ao aspecto social, a maioria dos participantes $(74,4 \%)$ respondeu que jogar videogame não interfere no seu relacionamento com seus amigos "reais"; $11 \%$ disseram que passaram a se relacionar mais com os amigos "virtuais" e $4,9 \%$ relataram que passam menos tempo com os amigos "reais". Comparando os dois grupos, notou-se que mais jogadores frequentes relataram que o uso de jogos eletrônicos interferiu em seus relacionamentos sociais $(p<0,01)$, e que passaram a relacionar-se mais com jogadores virtuais após começarem a prática de jogos eletrônicos $(p<0,05)$ do que jogadores ocasionais.

Quanto às atividades de lazer dos estudantes, as mais frequentes foram: "Navegar na internet" (77,1\%, diariamente) e "Ler ou ouvir música" (68,7\% diariamente), sendo que a maioria dos participantes afirma que sai com os amigos uma vez ou mais por semana $(63,4 \%)$ e grande parte afirmou que pratica esportes uma vez ou mais por semana $(48,2 \%)$.

A média da pontuação na escala PVP foi de 2,55 ( $D P=1,74)$, sendo que a maioria dos jogadores apresentou o escore de até 3 pontos $(74,4 \%)$ e 15,8\% dos participantes tiveram o escore de 5 ou mais. Quanto à frequência de jogo, observa-se que a média de escore obtido pelos jogadores ocasionais foi de 2,16 $(D P=1,72)$ e a dos jogadores frequentes foi de 3,12 (DP=1,63). Essa diferença foi estatisticamente significativa $(p<0,01)$.

A Tabela 3 apresenta a frequência com que cada item da PVP foi assinalado.

Procurando verificar se jogadores que preencheram mais itens da escala PVP se diferenciavam dos outros quanto a outras variáveis pesquisadas, observou-se que os denominados prováveis jogadores dependentes de videogame se diferenciaram dos jogadores sociais quanto às seguintes va- riáveis: jogar mais jogos de estratégia $(p<0,05)$, jogar mais RPG $(p<0,05)$, jogar pelo prazer no desafio de superar etapas $(p<0,05)$ e pela possibilidade de jogar com amigos, sejam eles virtuais $(p<0,05)$ ou reais $(p<0,05)$. Nas questões da escala PVP, chamou atenção o fato de um único item não distinguir jogadores sociais dos prováveis dependentes de videogame: o item VI relativo à necessidade de jogar novamente diante de uma derrota ou incapacidade de alcançar o objetivo desejado ( $p>0,1$ ), que foi assinalado por $56,8 \%$ da amostra.

\section{DISCUSSÃO}

No presente estudo observou-se que jogos eletrônicos, de videogame, computador e/ou internet foram praticados pela maioria dos estudantes universitários pesquisados, sugerindo que o padrão encontrado em crianças em idade escolar ${ }^{4}$ e em adolescentes ${ }^{6}$ parece persistir depois da adolescência, não estando relacionado à idade dos participantes. Jogos eletrônicos parecem fazer parte do cotidiano dessa amostra de estudantes, que joga preferencialmente jogos de ação e estratégia, em casa e à noite, e refere praticar a atividade para relaxar, se distrair, e pelo prazer no desafio de superar etapas, além do prazer de jogar com amigos. Dentre as diversas atividades de lazer praticadas, observouse que navegar na internet passou a ser atividade diária, em detrimento de atividades como assistir TV. Observou-se também que jogar jogos eletrônicos foi mais popular entre os homens do que entre mulheres, dado que coincide com outros estudos ${ }^{2,3,16}$. Os estudantes dividiram-se em duas frequências distintas de jogo: jogadores ocasionais e jogadores frequentes, tendo sido possível observar diferenças significativas entre os grupos, sugerindo a possibilidade de perfis distintos de envolvimento com jogos eletrônicos. Essas diferenças talvez apontem para fatores de risco para dependência de jogo eletrônico.

Tabela 3. Distribuição de respostas de estudantes universitários na escala PVP de acordo com frequência de jogo

\begin{tabular}{|c|c|c|c|c|c|c|c|}
\hline & \multicolumn{2}{|c|}{$\begin{array}{c}\text { Ocasionais } \\
(n=37)\end{array}$} & \multicolumn{2}{|c|}{$\begin{array}{l}\text { Frequentes } \\
(n=46)\end{array}$} & \multicolumn{2}{|c|}{$\begin{array}{l}\text { Total } \\
(\mathrm{n}=83)\end{array}$} & \multirow[t]{2}{*}{ p } \\
\hline & $\mathrm{n}$ & $\%$ & n & $\%$ & n & $\%$ & \\
\hline I - Quando não estou jogando jogos eletrônicos, fico pensando nos jogos (relembrando jogadas, planejando o próximo jogo etc.) & 5 & 14,3 & 23 & 50,0 & 28 & 34,6 & 0,001 \\
\hline II - Tenho gasto um tempo cada vez maior jogando. & 2 & 5,7 & 3 & 6,5 & 5 & 6,2 & 1,000 \\
\hline III - Já tentei controlar, diminuir ou parar de jogar, ou normalmente jogo por um período mais longo do que havia planejado. & 3 & 8,6 & 23 & 50,0 & 26 & 32,1 & 0,000 \\
\hline IV - Quando eu não posso jogar, fico inquieto ou irritado. & 0 & 0,0 & 5 & 10,9 & 5 & 6,2 & 0,066 \\
\hline V - Quando estou mal, isto é, nervoso, triste ou bravo, ou quando estou com problemas, eu jogo mais frequentemente. & 5 & 14,3 & 13 & 28,3 & 18 & 22,2 & 0,180 \\
\hline VI - Quando perco um jogo ou não alcanço os resultados desejados, preciso jogar de novo para alcançar meus objetivos. & 17 & 48,6 & 29 & 63,0 & 46 & 56,8 & 0,258 \\
\hline VII - Às vezes jogo escondido de outras pessoas, isto é, de meus pais, amigos, professores... & 1 & 2,9 & 4 & 8,7 & 5 & 6,2 & 0,383 \\
\hline $\begin{array}{l}\text { VIII - Para jogar jogos eletrônicos, já fiz pelo menos uma das seguintes coisas: faltei em aulas ou no trabalho, menti, roubei, } \\
\text { discuti com alguém. }\end{array}$ & 5 & 14,3 & 10 & 21,7 & 15 & 18,5 & 0,565 \\
\hline $\begin{array}{l}\text { IX - Devido a jogar jogos eletrônicos já fiz pelo menos uma das seguintes coisas: fiz menos ou deixei de fazer lição de casa, trabalhos na } \\
\text { faculdade, deixei de comer, fui dormir muito tarde, passei menos tempo com amigos e família. }\end{array}$ & 21 & 60,0 & 41 & 89,1 & 62 & 76,5 & 0,003 \\
\hline
\end{tabular}


Um número significativamente superior de estudantes classificados como jogadores frequentes afirmou jogar mais tempo por sessão, preferir jogos de estratégia, RPG e esportes, jogar pelo desafio em superar etapas e pela possiblidade de jogar com amigos do que os classificados como jogadores ocasionais. Jogos do tipo Role Playing Games (RPG) e os Massive Multiplayer On-line Role-Playing Game (MMORPG), que permitem vários jogadores simultaneamente, têm chamado a atenção pelo fato de serem populares entre jogadores que apresentam uso excessivo de jogos eletrônicos ${ }^{12}$. Acredita-se que MMORPGs possuam alto potencial de provocar dependência por promoverem uma contingência de condicionamento operante intermitente, uma vez que os acontecimentos do jogo são imprevisíveis; por serem jogados on-line, permitindo a formação de laços sociais, e promovendo, portanto, reforçamento social; e ainda por apresentar propriedades imersivas, ou seja, os jogadores adentram um ambiente alternativo ${ }^{17}$. Outro aspecto que parece contribuir para esse potencial adictivo dos jogos eletrônicos, presente em jogos de RPG e também em jogos de estratégia, é o componente de avanço, ou seja, a necessidade de progredir e superar etapas ${ }^{14}$. Coincidindo com esses dados, no presente estudo houve correlação positiva entre entrevistados que afirmaram jogar jogos de RPG e estratégia e aqueles que afirmaram ser atraídos aos jogos pelo prazer no desafio de superar etapas.

A possibilidade de fazer amigos também diferenciou os estudantes: mais jogadores classificados como frequentes relataram que jogar videogame interfere nos seus relacionamentos sociais e que se relacionam mais com amigos virtuais do que jogadores ocasionais. Cerca de $5 \%$ dos jogadores relataram que passam menos tempo com os amigos "reais", indo ao encontro da hipótese que sugere que esses jogos podem substituir relacionamentos sociais e encorajar o isolamento ${ }^{18}$. No entanto, a maioria dos participantes respondeu que a prática de jogos eletrônicos não interfere no seu relacionamento com seus amigos "reais"; corroborando hipótese de que possibilitariam novas formas de socialização'.

Apesar de o presente estudo ter sido realizado com população universitária, a média da pontuação na escala $\operatorname{PVP}(M=2,55, D P=1,74)$ assemelhou-se à encontrada entre adolescentes $^{4}(M=2,70, D P=1,94$ para meninos e $M=1,50$ e $\mathrm{DP}=1,52$ para meninas) e foi inferior à encontrada por outro estudo on-line ${ }^{14}$, no qual se aplicou a escala em uma amostra de participantes de diversas nacionalidades entre 18 e 60 anos $(M=4,17, D P=2,19)$.

Os itens significativamente mais assinalados por jogadores frequentes do que ocasionais na escala PVP sugerem que a atividade de jogar está interferindo na vida do jogador e que talvez pudessem ser considerados sinais de alerta para atividade de risco. Quando se comparou estudantes que preencheram mais da metade da escala PVP, os prováveis dependentes de videogame, com os demais, observou-se que preencheram mais todos os itens, exceto um, evidenciando diferente envolvimento com a atividade. $\mathrm{O}$ item VI, que não diferenciou os grupos, referente à necessidade de jogar novamente diante de uma derrota ou incapacidade de alcançar o objetivo desejado, parece comum à maioria dos jogadores. Comparando esses prováveis dependentes de videogame com os demais, observou-se que jogam mais jogos de estratégia e RPG, e que um número significativamente superior relata jogar pelo prazer do desafio, e pela possibilidade de jogar com amigos reais e virtuais, sugerindo um perfil semelhante ao encontrado quando se comparou os estudantes pela frequência com que jogam.

Pode ser considerado alto o índice de estudantes que preencheram mais da metade da escala PVP, sugerindo problemas relacionados ao uso excessivo de jogos eletrônicos nessa amostra. No entanto, o item VI da escala pode ter contribuído para o alto índice. Dessa maneira, estudos futuros destinados a estabelecer um ponto de corte para a escala PVP devem avaliar esse item através de análise fatorial. Além disso, o uso de critérios retirados do DSM em um contexto de dependência comportamental vem sendo discutido e há autores que opõem a noção de dependência comportamental à de alto envolvimento, que seria uma alta dedicação a alguma atividade, sem que isso trouxesse consequências negativas ${ }^{20}$. No presente estudo, pode-se constatar alto envolvimento com o jogo por uma parcela da amostra, mas é preciso ter cautela para classificar os estudantes como dependentes a partir da pontuação na escala PVP. São estudantes que frequentam o campus universitário e relatam ter diversas atividades de lazer, além do que não foram avaliados aspectos importantes como desempenho acadêmico. Fatores importantes que denunciam excesso e/ou perda de controle sobre o comportamento, como jogar mais de 4 horas diárias e precisar ser interrompido por terceiros para parar de jogar, foram citados por cerca de 5\% dos entrevistados, porcentagem bastante inferior aos 15,8\% que preencheram mais da metade da escala, explicitando a complexidade da questão e a necessidade de instrumentos mais precisos.

Finalmente, ao investigar a influência de jogos violentos sobre o comportamento do jogador, no presente estudo, a maioria afirmou que isso não interfere em seu estado emocional, mas 7,4\% dos entrevistados relataram sentir-se mais agressivos, o que deve ser mais bem investigado em estudos futuros.

Os resultados apresentados devem ser interpretados com algumas limitações. A amostra estudada é bastante particular (estudantes de uma universidade pública de São Paulo que têm a possibilidade de estudar durante o dia), não sendo uma amostra estratificada. Também foi composta por jovens que foram abordados no campus, ou seja, que estavam fora de suas casas, em ambiente acadêmico, o 
que já seria um viés de exclusão de sujeitos com problemas mais graves de socialização. Além disso, algumas diferenças não puderam ser confirmadas devido ao reduzido tamanho da amostra. Outra limitação importante a ser considerada advém de se tratar de pesquisa baseada em relato dos participantes em questionário de autopreenchimento. Estudos futuros deverão incluir amostra maior e representativa, visando um perfil dos estudantes universitários brasileiros.

\section{CONCLUSÃO}

Os jogos eletrônicos, de forma geral, parecem ser uma prática que faz parte do cotidiano dos estudantes que compuseram a amostra entrevistada. Apesar de a maioria jogar por lazer, estudantes que jogam com frequência acima de uma vez por semana apresentaram mais indícios de problemas relacionados ao jogo e cerca de 15\% da amostra preencheu mais da metade da escala PVP, indicando consequências adversas decorrentes desse hábito. Além disso, cerca de 5\% relataram só parar de jogar quando interrompidos por terceiros, normalmente jogar mais de 4 horas por sessão e se relacionar mais com amigos virtuais, sugerindo maior envolvimento com a atividade. Faz-se necessária uma análise mais aprofundada dos possíveis fatores de risco associados ao uso excessivo dos jogos eletrônicos, bem como o aprimoramento de um instrumento para o diagnóstico do uso patológico de jogos eletrônicos.

\section{AGRADECIMENTOS}

Gostaríamos de expressar nossos agradecimentos às colegas de graduação Karen Cristina Fachini Furlan e Lúcia Kaori Masumoto, por sua colaboração na elaboração do questionário e coleta de dados. Também somos gratos ao Instituto de Psicologia da Universidade de São Paulo, pela permissão de uso de recursos técnicos disponíveis no laboratório de Psicofarmacologia para a elaboração do questionário e análise dos resultados.

\section{REFERÊNCIAS}

1. Soper WB, Miller MJ. Junk times junkies: an emerging addiction among students. School Counselor. 1983;31(1):40-43

2. Phillips CA, Rolls S, Rouse A, Griffiths MD. Home video game playing in schoolchildren: a study of incidence and patterns of play. Journal of Adolescence. 1995;18:687-91.

3. Griffiths MD, Hunt N. Dependence on computer games by adolescents. Psychological Reports. 1998;82:475-80.

4. Morán RMB, Salguero RAT. Measuring problem video game playing in adolescents. Blackwell Synergy-Addiction. 2002;97(12):1601-6.

5. Psychological Science Agenda - American Psychological Association. Violent Video Games: Myths, Facts and Unanswered Questions. [acesso em 16 dez 2008] Disponível em: http://www.psychology.iastate.edu/faculty/caa/abstracts/2000-2004/03A2.pdf.

6. Cover R. Gaming (Ad)diction: Discourse, Identity, Time and Play in the Production of the Gamer Addiction Myth. Game Studies [periódico na Internet]. 2006 Dez; 6(1). [acesso em 14 abr 2008] Disponível em: http://gamestudies.org/0601/articles/cover.

7. Rossetti C B et al. Jogos eletrônicos violentos e estratégias de resolução de conflitos de jovens da cidade de Vitória. Pesquisas e Práticas Psicossociais. 2007;2(1).

8 Chiu S, Lee J, Huang D. Video game addiction in children and teenagers in Taiwan. CyberPsychology \& Behavior. 2004;7(5):571-81.

9. Buchman DD, Funk JB. Video and computer games in the 90 s: children's time commitment \& game preference. Children Today. 1996;24:12-5.

10. Selnow G. Playing videogames: the electronic friend. Journal of Communication. 1984; 34(2):148-56

11. Morahan-Martin J, Schumacher P. Incidence and correlates of pathological internet use among college students. Comp Human Behav. 2000;16:13-29.

12. Brian DNG, Wiemer-Hastings P. Addiction to the internet and online gaming. CyberPsychology \& Behavior. 2005;8(2):110-13.

13. Abreu CN, Karan RG, Góes DS, Spritzer DT. Dependência de internet e jogos eletrônicos: uma revisão. Rev Bras Psiquiatr. 2008,30(2): 156-67.

14. Loton D. Problem Video Game Playing, Self Esteem and Social Skills: An Online Study [monografia na internet]. Victoria University; 2007. [acesso em 16 dez 2008] Disponível em: http://eprints.vu.edu.au/687/1/Problemvideogameplaying.pdf.

15. Gee JP. What video games have to teach us about learning and literacy. New York: Palgrave Macmillan; 2003.

16. Fromme J. Computer game as a part of children's culture. Game Studies. 2003;3:1.

17. Charlton JP, Danforth IDW. Distinguishing addiction and high engagement in the context of online game playing. Computers in Human Behaviour. 2007;3:1531-48.

18. Barnett MA, Vitaglione GD, Harper KKG, Quackenbush SW, Steadman LA, Valdez BS. Late adolescents' experiences with and attitudes towards videogames. Journal of Applied Social Psychology. 1997;27(15):1316-34.

19. Durkin K, Barber B. Not so doomed: computer game play and positive adolescent development. Applied Developmental Psychology. 2002;23:373-92.

20. Charlton JP, Birkett PE. The development and validation of the Computer Apathy and Anxiety Scale. Journal of Educational Computing Research. 1995;13(1):41-59. 